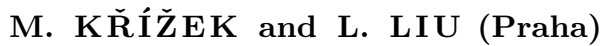

\title{
ON A COMPARISON PRINCIPLE FOR A QUASILINEAR ELLIPTIC BOUNDARY VALUE PROBLEM OF A NONMONOTONE TYPE
}

Abstract. A nonlinear elliptic partial differential equation with the Newton boundary conditions is examined. We prove that for greater data we get a greater weak solution. This is the so-called comparison principle. It is applied to a steady-state heat conduction problem in anisotropic magnetic cores of large transformers.

1. Introduction. Comparison and maximum principles are important features of second order elliptic equations that distinguish them from higher order equations and systems of equations. In this paper we deal with a quasilinear elliptic problem whose classical formulation reads:

Find $u \in C^{1}(\bar{\Omega})$ such that $\left.u\right|_{\Omega} \in C^{2}(\Omega)$ and

$$
\begin{aligned}
-\operatorname{div}(\mathcal{A}(\cdot, u) \operatorname{grad} u) & =f & & \text { in } \Omega, \\
\alpha u+n^{T} \mathcal{A}(\cdot, u) \operatorname{grad} u & =g & & \text { on } \partial \Omega,
\end{aligned}
$$

where $\Omega \subset \mathbb{R}^{d}, d \in\{1,2, \ldots\}$, is a bounded domain with a Lipschitz continuous boundary, $n=\left(n_{1}, \ldots, n_{d}\right)^{T}$ is the outward unit normal to $\partial \Omega$, $\mathcal{A}=\left(a_{i j}\right)_{i, j=1}^{d}$ is a uniformly positive definite matrix and $\alpha \geq 0$. Let the functions $\mathcal{A}, \alpha, f$ and $g$ be sufficiently smooth for the time being (precise assumptions on these functions are given in Section 2).

The problem (1.1)-(1.2) describes a steady-state heat conduction in nonlinear inhomogeneous anisotropic media. The unknown function $u$ represents the temperature, $\mathcal{A}$ is the matrix of heat conductivities, $\alpha$ is the heat transfer coefficient, $f$ is the density of volume heat sources and $g$ is the density of surface heat sources. The existence and uniqueness of $u$ is studied in [16] (and in $[5,6,12,13]$ for similar problems with other boundary conditions).

1991 Mathematics Subject Classification: Primary 35J60.

Key words and phrases: nonlinear boundary value problem, comparison principle, anisotropic heat conduction. 
The main aim of this paper is to show the following comparison principle:

$$
f_{1} \leq f_{2} \& g_{1} \leq g_{2} \Rightarrow u_{1} \leq u_{2}
$$

where $u_{i}$ is a weak solution of the problem (1.1)-(1.2) corresponding to the densities $f_{i}$ and $g_{i}$ of heat sources for $i=1,2$. Our method is completely different from that used in $[5,10,23]$, where the classical solution $u \in C^{2}(\Omega)$ is considered. Moreover, our assumptions on the matrix $\mathcal{A}(\cdot, u)$ are not covered by $[5,10,23]$. Note that the comparison principle (1.3) for linear problems (i.e., when $\mathcal{A}$ is independent of $u$ ) is a consequence of the weak maximum principle (see, e.g., [10, p. 32, 207]). For more information about maximum principles we refer to $[21]$.

2. Weak formulation and existence. To state a weak formulation of problem (1.1)-(1.2) we assume that $\mathcal{A}=\mathcal{A}(\cdot, \cdot)$ and $\alpha=\alpha(\cdot)$ are bounded measurable functions,

$$
\underset{x, \xi, i, j}{\operatorname{ess} \sup }\left|a_{i j}(x, \xi)\right| \leq C, \quad \operatorname{essup}|\alpha(s)| \leq C,
$$

where $x \in \Omega, \xi \in \mathbb{R}^{1}, i, j \in\{1, \ldots, d\}$ and $s \in \partial \Omega$. The components $a_{i j}$ are assumed to be Lipschitz continuous with respect to the second variable, i.e., there exists $C_{L}>0$ such that for all $\zeta, \xi \in \mathbb{R}^{1}$ and almost all $x \in \Omega$ we have

$$
\left|a_{i j}(x, \zeta)-a_{i j}(x, \xi)\right| \leq C_{L}|\zeta-\xi|, \quad i, j=1, \ldots, d .
$$

Further, let there exist $C_{0}>0$ such that for almost all $x \in \Omega$,

$$
C_{0} \eta^{T} \eta \leq \eta^{T} \mathcal{A}(x, \xi) \eta \quad \forall \xi \in \mathbb{R}^{1} \forall \eta \in \mathbb{R}^{d}
$$

and let $0 \leq \alpha(s)$ for almost all $s \in \partial \Omega$. To guarantee the existence of $u$, we moreover assume that there exists a constant $\alpha_{0}>0$ and a nonempty relatively open subset $\Gamma \subset \partial \Omega$ such that

$$
\alpha(s) \geq \alpha_{0}
$$

for almost all $s \in \Gamma$. Recall that the boundary condition (1.2) is called the Newton boundary condition. It is called the Neumann boundary condition at those parts of $\partial \Omega$ where $\alpha=0$.

Finally, let $f \in L^{2}(\Omega), g \in L^{2}(\partial \Omega)$ and $V=H^{1}(\Omega)$, where $H^{1}(\Omega)$ is the Sobolev space of functions whose first generalized derivatives belong to $L^{2}(\Omega)$.

For simplicity, a possible dependence of $\mathcal{A}$ on $x$ is not explicitly indicated in what follows. Set

$$
\begin{aligned}
a(y ; w, v) & =(\mathcal{A}(y) \operatorname{grad} w, \operatorname{grad} v)_{0, \Omega}+\langle\alpha w, v\rangle_{0, \partial \Omega}, & & y, w, v \in V, \\
F(v) & =(f, v)_{0, \Omega}+\langle g, v\rangle_{0, \partial \Omega}, & & v \in V,
\end{aligned}
$$

where $(\cdot, \cdot)_{0, \Omega}$ and $\langle\cdot, \cdot\rangle_{0, \partial \Omega}$ stand for the usual scalar products in $L^{2}(\Omega)$ and $L^{2}(\partial \Omega)$, respectively. Since $\mathcal{A}$ and $\alpha$ are bounded by (2.1), we observe that 
both the terms on the right hand side of $(2.5)$ are finite, i.e., $a(\cdot ; \cdot, \cdot)$ is well defined. Throughout the paper, the symbol $\|\cdot\|_{k, \Omega}$ is used for the norm in the product Sobolev space $\left(H^{k}(\Omega)\right)^{q}$ for $k \in\{0,1, \ldots\}$ and $q \in\{1,2, \ldots\}$.

Suppose that some $u$ satisfy (1.1)-(1.2). Multiplying (1.1) by an arbitrary test function $v \in V$ and then integrating over $\Omega$, we arrive, by (1.2) and the Green formula, at the following definition:

Definition 2.1. A function $u \in V$ is said to be a weak solution of the problem (1.1)-(1.2) if

$$
a(u ; u, v)=F(v) \quad \forall v \in V .
$$

Next we present several remarks concerning the existence of the weak solution.

R e mark 2.2. The well-known Kirchhoff transformation (see $[2,9,17]$ ), which changes the nonlinear problem to a linear one, can be applied in the case of isotropic nonlinear media, i.e., when $\mathcal{A}$ is a scalar function. However, it cannot be applied to prove the existence of $u$ in the case of anisotropic nonlinear media, in general. For instance, in examining a temperature field in the magnetic circuit of a transformer (see Figure 3), nonlinear temperature dependencies of heat conductivities across and along lamination differ. The associated $3 \times 3$ matrix $\mathcal{A}$ of heat conductivities is diagonal and such that $a_{22} \neq a_{11}=a_{33}$. The temperature dependencies of the diagonal entries differ in such a way that the types of nonlinearity in the $x_{1}$ and $x_{2}$ directions are different (see [16]).

R e mark 2.3. To prove the existence of a weak solution $u \in V$ we cannot apply the Minty-Browder theorem for monotone operators (cf. [9]), since our problem does not in general lead to a monotone operator (see [13, p. 171] for a one-dimensional example).

Remark 2.4. The problem of Definition 2.1 cannot be transformed to the minimization of some functional, since the associated operator $\mathbb{A}$ is not in general a potential operator (see [12, p. 87] for a one-dimensional example). The well-known symmetry conditions from [20, p. 41] are not satisfied.

R e m a r k 2.5. We observe that there exists a constant $C_{0}>0$ such that

$$
C_{0}\|v\|_{1, \Omega}^{2} \leq a(y ; v, v) \quad \forall y, v \in V .
$$

This inequality is a direct consequence of (2.3)-(2.5) and the following Friedrichs' inequality (see [19, p. 20]):

$$
\|v\|_{1, \Omega}^{2} \leq C\left(\|\operatorname{grad} v\|_{0, \Omega}^{2}+\|v\|_{0, \Gamma}^{2}\right) \quad \forall v \in V .
$$

Using (2.5), (2.6), the boundedness of $\mathcal{A}, \alpha$ (see (2.1)) and the trace theorem (see [19, p. 84]), it is not difficult to verify that

$$
|a(y ; w, v)| \leq C\|w\|_{1, \Omega}\|v\|_{1, \Omega} \quad \forall y, w, v \in V,
$$




$$
|F(v)| \leq C\|v\|_{1, \Omega} \quad \forall v \in V .
$$

TheOREm 2.6. Let (2.1)-(2.4) hold. Then there exists a weak solution of the problem (1.1)-(1.2).

For the proof see [13]. The weak solution is obtained as a weak limit of Galerkin approximations. The proof is based on the properties (2.8)-(2.10), the well-known Brouwer theorem $[8,16]$ and the density theorem of [3].

R e mark 2.7. From (2.8), (2.7), (2.6) and the trace theorem we have

$$
\begin{aligned}
C_{0}\|u\|_{1, \Omega}^{2} & \leq a(u ; u, u)=(f, u)_{0, \Omega}+\langle g, u\rangle_{0, \partial \Omega} \\
& \leq C\left(\|f\|_{0, \Omega}+\|g\|_{0, \partial \Omega}\right)\|u\|_{1, \Omega} .
\end{aligned}
$$

Hence, there exists a constant $C>0$ (independent of the data $f, g$ ) such that

$$
\|u\|_{1, \Omega} \leq C\left(\|f\|_{0, \Omega}+\|g\|_{0, \partial \Omega}\right) .
$$

This means that we can estimate the norm $\|u\|_{1, \Omega}$ by the "data". We observe from (2.11) that for vanishing data the weak solution $u$ is unique.

3. The comparison principle. In this section we prove the uniqueness of $u$ for any $f \in L^{2}(\Omega)$ and $g \in L^{2}(\partial \Omega)$. This result will be a consequence of the following comparison principle.

TheOrem 3.1. Let (2.1)-(2.4) hold and let $u_{1}, u_{2} \in V$ be two weak solutions of the problem (2.7) corresponding to $f_{1}, f_{2} \in L^{2}(\Omega)$ and $g_{1}, g_{2} \in$ $L^{2}(\partial \Omega)$, respectively. Assume that

$$
f_{1} \geq f_{2} \quad \text { a.e. in } \Omega
$$

and

$$
g_{1} \geq g_{2} \quad \text { a.e. in } \partial \Omega .
$$

Then $u_{1} \geq u_{2}$ a.e. in $\Omega$.

Proof. Let $f_{1} \geq f_{2}, g_{1} \geq g_{2}$ and let $u_{1}, u_{2}$ be the corresponding weak solutions. Put $\Omega_{0}=\left\{x \in \Omega \mid u_{1}(x)<u_{2}(x)\right\}$ and assume, on the contrary, that

$$
\text { meas } \Omega_{0}>0 \text {. }
$$

Let $\varepsilon>0$ be arbitrary and let us define (see Figure 1)

$$
\begin{aligned}
& \Omega_{\varepsilon}=\left\{x \in \Omega_{0} \mid u_{2}-u_{1}>\varepsilon\right\}, \\
& v_{\varepsilon}= \begin{cases}\min \left(\varepsilon, u_{2}-u_{1}\right) & \text { in } \Omega_{0}, \\
0 & \text { in } \mathbb{R}^{d} \backslash \Omega_{0} .\end{cases}
\end{aligned}
$$

We show that $v_{\varepsilon}$ can be applied in (2.7) as a test function. Since $u_{2}-u_{1} \in$ $V$, the positive part $\left(u_{2}-u_{1}\right)^{+}$also lies in $V$. This is due to the fact that $v \mapsto v^{+}$is a continuous mapping from $H^{1}(\Omega)$ to $H^{1}(\Omega)$ (see, e.g., [11, 


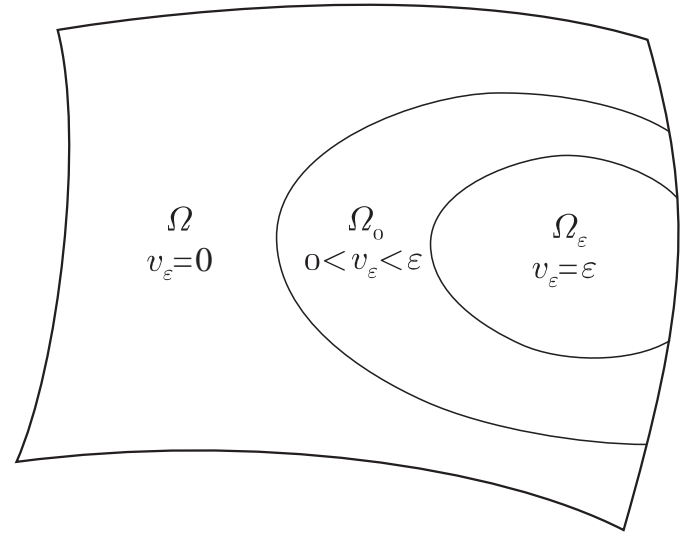

Fig. 1

p. 29]). The mapping $v \mapsto|v|=v^{+}+v^{-}$is continuous as well. Therefore, the equality $\min (a, b)=\frac{1}{2}(a+b-|a-b|)$ implies that

$$
v_{\varepsilon}=\min \left(\varepsilon,\left(u_{2}-u_{1}\right)^{+}\right) \in V .
$$

Thus, by (2.7), we may write

$$
\begin{aligned}
\left(\mathcal{A}\left(u_{i}\right) \operatorname{grad} u_{i}, \operatorname{grad} v_{\varepsilon}\right)_{0, \Omega} & +\left\langle\alpha u_{i}, v_{\varepsilon}\right\rangle_{0, \partial \Omega} \\
& =\left(f_{i}, v_{\varepsilon}\right)_{0, \Omega}+\left\langle g_{i}, v_{\varepsilon}\right\rangle_{0, \partial \Omega}, \quad i=1,2 .
\end{aligned}
$$

Since $v_{\varepsilon} \geq 0$ and $\alpha \geq 0$, we have, by (3.5),

$$
\alpha\left(u_{1}-u_{2}\right) v_{\varepsilon} \leq 0 \quad \text { on } \partial \Omega \text {. }
$$

From (2.3), (3.5), (3.7), (3.8), (3.1) and (3.2) we obtain

$$
\begin{aligned}
& C_{0}\left\|\operatorname{grad} v_{\varepsilon}\right\|_{0, \Omega}^{2} \\
& \leq\left(\mathcal{A}\left(u_{1}\right) \operatorname{grad} v_{\varepsilon}, \operatorname{grad} v_{\varepsilon}\right)_{0, \Omega} \\
&=\left(\mathcal{A}\left(u_{1}\right) \operatorname{grad}\left(u_{2}-u_{1}\right), \operatorname{grad} v_{\varepsilon}\right)_{0, \Omega_{0} \backslash \Omega_{\varepsilon}} \\
&=\left(\mathcal{A}\left(u_{1}\right) \operatorname{grad} u_{2}-\mathcal{A}\left(u_{1}\right) \operatorname{grad} u_{1}, \operatorname{grad} v_{\varepsilon}\right)_{0, \Omega} \\
&=\left(\mathcal{A}\left(u_{1}\right) \operatorname{grad} u_{2}-\mathcal{A}\left(u_{2}\right) \operatorname{grad} u_{2}, \operatorname{grad} v_{\varepsilon}\right)_{0, \Omega} \\
&+\left\langle\alpha\left(u_{1}-u_{2}\right), v_{\varepsilon}\right\rangle_{0, \partial \Omega}+\left(f_{2}-f_{1}, v_{\varepsilon}\right)_{0, \Omega}+\left\langle g_{2}-g_{1}, v_{\varepsilon}\right\rangle_{0, \partial \Omega} \\
& \leq\left(\left(\mathcal{A}\left(u_{1}\right)-\mathcal{A}\left(u_{2}\right)\right) \operatorname{grad} u_{2}, \operatorname{grad} v_{\varepsilon}\right)_{0, \Omega} .
\end{aligned}
$$

The last scalar product can be further estimated by (3.4), (3.5), the CauchySchwarz inequality and (2.2) as follows: 


$$
\begin{aligned}
\left(\left(\mathcal{A}\left(u_{1}\right)-\right.\right. & \left.\left.\mathcal{A}\left(u_{2}\right)\right) \operatorname{grad} u_{2}, \operatorname{grad} v_{\varepsilon}\right)_{0, \Omega_{0} \backslash \Omega_{\varepsilon}} \\
& \leq\left\|\left(\mathcal{A}\left(u_{1}\right)-\mathcal{A}\left(u_{2}\right)\right) \operatorname{grad} u_{2}\right\|_{0, \Omega_{0} \backslash \Omega_{\varepsilon}}\left\|\operatorname{grad} v_{\varepsilon}\right\|_{0, \Omega_{0} \backslash \Omega_{\varepsilon}} \\
& \leq \varepsilon C_{L} d^{2}\left\|\operatorname{grad} u_{2}\right\|_{0, \Omega_{0} \backslash \Omega_{\varepsilon}}\left\|\operatorname{grad} v_{\varepsilon}\right\|_{0, \Omega_{0} \backslash \Omega_{\varepsilon}} .
\end{aligned}
$$

Combining (3.9) and (3.10), we obtain

$$
\left\|\operatorname{grad} v_{\varepsilon}\right\|_{0, \Omega} \leq \varepsilon C\left\|\operatorname{grad} u_{2}\right\|_{0, \Omega_{0} \backslash \Omega_{\varepsilon}} .
$$

According to (3.5) and (2.4),

$$
0 \leq v_{\varepsilon} \leq\left(u_{2}-u_{1}\right)^{+}=u_{2}-u_{1} \leq \frac{1}{\alpha_{0}}\left(\alpha u_{2}-\alpha u_{1}\right) \quad \text { on } \Gamma \cap \bar{\Omega}_{0},
$$

and thus (since $v_{\varepsilon}=0$ on $\Gamma \backslash \bar{\Omega}_{0}$ )

$$
v_{\varepsilon}^{2} \leq \frac{1}{\alpha_{0}}\left(\alpha u_{2}-\alpha u_{1}\right) v_{\varepsilon} \quad \text { on } \Gamma \text {. }
$$

Moreover, by (3.9),

$$
\begin{aligned}
-\left\langle\alpha u_{1}-\alpha u_{2}, v_{\varepsilon}\right\rangle_{0, \partial \Omega}+C_{0}\left\|\operatorname{grad} v_{\varepsilon}\right\|_{0, \Omega}^{2} & \\
& \leq\left(\left(\mathcal{A}\left(u_{1}\right)-\mathcal{A}\left(u_{2}\right)\right) \operatorname{grad} u_{2}, \operatorname{grad} v_{\varepsilon}\right)_{0, \Omega} .
\end{aligned}
$$

Consequently, Friedrichs' inequality, (3.12), (3.10) and (3.11) imply that

$$
\begin{aligned}
\left\|v_{\varepsilon}\right\|_{0, \Omega}^{2} & \leq C_{1}\left(\left\|v_{\varepsilon}\right\|_{0, \Gamma}^{2}+\left\|\operatorname{grad} v_{\varepsilon}\right\|_{0, \Omega}^{2}\right) \\
& \leq C_{2}\left(\left\langle\alpha u_{2}-\alpha u_{1}, v_{\varepsilon}\right\rangle_{0, \Gamma}+\left\|\operatorname{grad} v_{\varepsilon}\right\|_{0, \Omega}^{2}\right) \\
& \leq C_{3}\left(\left\langle\alpha u_{2}-\alpha u_{1}, v_{\varepsilon}\right\rangle_{0, \partial \Omega}+C_{0}\left\|\operatorname{grad} v_{\varepsilon}\right\|_{0, \Omega}^{2}\right) \\
& \leq C_{3}\left(\left(\mathcal{A}\left(u_{1}\right)-\mathcal{A}\left(u_{2}\right)\right) \operatorname{grad} u_{2}, \operatorname{grad} v_{\varepsilon}\right)_{0, \Omega} \\
& \leq \varepsilon C_{4}\left\|\operatorname{grad} u_{2}\right\|_{0, \Omega_{0} \backslash \Omega_{\varepsilon}}\left\|\operatorname{grad} v_{\varepsilon}\right\|_{0, \Omega_{0} \backslash \Omega_{\varepsilon}} \\
& \leq \varepsilon^{2} C_{5}\left\|\operatorname{grad} u_{2}\right\|_{0, \Omega_{0} \backslash \Omega_{\varepsilon}}^{2} .
\end{aligned}
$$

From this, (3.5), the facts that $u_{2}$ is fixed and $\Omega_{\varepsilon} \subset \Omega_{0}$, we arrive at

$$
\begin{aligned}
\operatorname{meas} \Omega_{\varepsilon} & =\varepsilon^{-2} \int_{\Omega_{\varepsilon}} \varepsilon^{2} d x \leq \varepsilon^{-2}\left\|v_{\varepsilon}\right\|_{0, \Omega}^{2} \\
& \leq C_{5}\left\|\operatorname{grad} u_{2}\right\|_{0, \Omega_{0} \backslash \Omega_{\varepsilon}}^{2} \rightarrow 0 \quad \text { as } \varepsilon \rightarrow 0,
\end{aligned}
$$

where $C_{5}>0$ is independent of $\varepsilon$. This, however, contradicts (3.3) and (3.4), since meas $\Omega_{\varepsilon} \rightarrow$ meas $\Omega_{0}$. Consequently, meas $\Omega_{0}=0$ and $u_{1} \geq u_{2}$ a.e. in $\Omega$.

Corollary 3.2. Let (2.1)-(2.4) hold. Then there exists at most one weak solution of the problem (1.1)-(1.2).

Proof. According to Theorem 3.1 we have $u_{1} \geq u_{2}$ and $u_{1} \leq u_{2}$ for $f_{1}=f_{2}$ and $g_{1}=g_{2}$. This proves the uniqueness of the weak solution $u$. 
Remark 3.3. Theorem 3.1 and its proof can be easily modified for Dirichlet boundary conditions or mixed Dirichlet-Newton boundary conditions. A proof of the uniqueness of the classical solution of the problem (1.1)-(1.2) is given in [5] for the Dirichlet boundary conditions and in [12] for mixed conditions. The uniqueness of the weak solution for the mixed nonlinear boundary conditions is proved also in [13] without application of the comparison principle. Other uniqueness theorems for general nonlinear problems with Dirichlet boundary conditions are given in $[1,14]$.

R e m a r k 3.4. There exist examples of nonunique solutions if the elliptic equation is not in the divergence form (see, e.g., [10, p. 209], [18, p. 178]). We can also get nonunique solutions of our divergence form problem (1.1)-(1.2) if the condition (2.2) is violated. To see this, we recall the following example due to J. Malý. Let $d=1, \Omega=(0,1)$ and consider two fixed real smooth functions $u_{1}, u_{2}$ such that $u_{1}<u_{2}$ on $(0,1), u_{1}(0)=u_{2}(0), u_{1}^{\prime}(0)=u_{2}^{\prime}(0)$, $u_{1}(1)=u_{2}(1), u_{1}^{\prime}(1)=u_{2}^{\prime}(1), u_{1}^{\prime} \geq 1$ and $u_{2}^{\prime} \geq 1$ (see Figure 2 ).

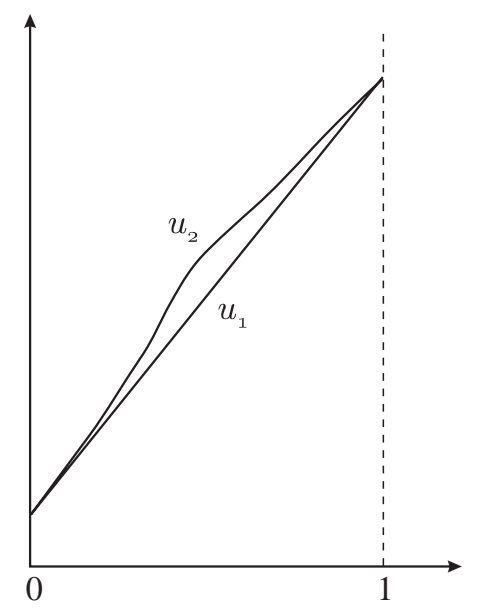

Fig. 2

Let us define a real function $\mathcal{A}$ on the graphs of $u_{1}$ and $u_{2}$ as follows:

$$
\mathcal{A}(x, \xi)=\frac{1}{u_{i}^{\prime}(x)} \quad \text { for } x \in[0,1], \xi=u_{i}(x), i=1,2 .
$$

Then by Tietze's extension theorem (see, e.g., [22, p. 422]) there exists a continuous extension (still denoted by $\mathcal{A}$ ) so that $\mathcal{A}(\cdot, \cdot): \Omega \times \mathbb{R}^{1} \rightarrow \mathbb{R}^{1}$ and (2.1) and (2.3) hold. We see that

$$
-\left(\mathcal{A}\left(x, u_{i}\right) u_{i}^{\prime}\right)^{\prime}=0 \quad \text { for } i=1,2,
$$

i.e., $u_{1}$ and $u_{2}$ are solutions of (1.1) with $f=0$ and the Newton boundary conditions (1.2) for $\alpha=1$ and $g=0$. However, in this case it is not difficult 
to check that $\mathcal{A}$ is not Lipschitz continuous (with respect to the second variable) near those points where $u_{1}$ and $u_{2}$ bifurcate. The condition (2.2) is thus essential to get the uniqueness.

Another one-dimensional example of nonunique solutions of a nonlinear elliptic boundary value problem is given in $[1$, p. 1163].

4. An application. A steady-state heat conduction problem defined by (1.1)-(1.2) describes a temperature distribution in large transformers. Their magnetic cores (consisting of iron sheets) are nonlinear orthotropic media the heat conductivities of which can be represented by a diagonal matrix $\mathcal{A}=\mathcal{A}(u)$. The temperature dependencies of the heat conductivity coefficients $a_{11}(u)$ and $a_{22}(u)$ across and along the lamination, respectively, differ substantially (see Remark 2.2).

Due to the symmetry of the magnetic core (see Figure 3 ), we can solve the problem (1.1)-(1.2) on a smaller domain, which will be denoted by $\Omega$.

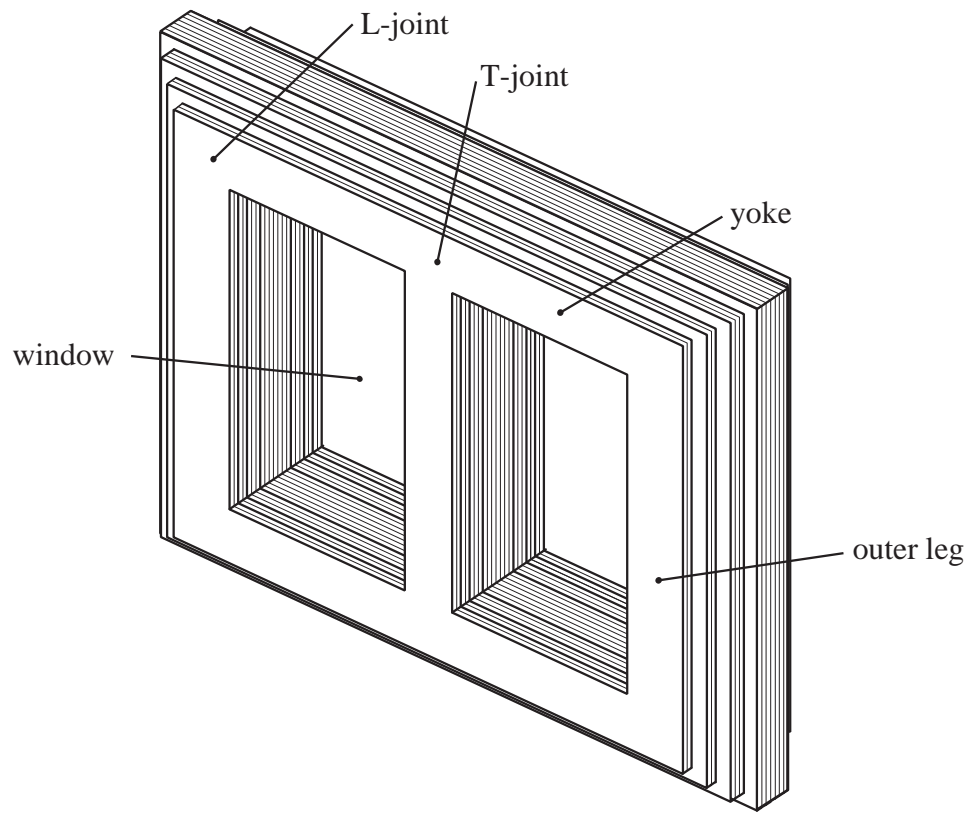

Fig. 3

We prescribe the homogeneous Neumann boundary conditions (i.e. $\alpha=0$ in (1.2)) at those parts of $\partial \Omega$ which correspond to planes of symmetry, and let $\alpha \geq \alpha_{0}>0$ be the heat transfer coefficient on the remaining part $\Gamma$ of the boundary $\partial \Omega$. This means that $\Gamma$ is that part of the boundary $\partial \Omega$ which is cooled by oil and $\partial \Omega \backslash \Gamma$ corresponds to all planes of symmetry. 
We have $g=\alpha u_{0}$ on $\Gamma$ (cf. (1.2)), where $u_{0}$ is the temperature of cooling oil, and $g=0$ on $\partial \Omega \backslash \Gamma$. The density $f$ of volume heat sources is positive due to the alternating electromagnetic field.

Note that the knowledge of the temperature distribution is very important to avoid a local overheating. If the temperature exceeds prescribed limits, the cooling oil starts to boil which may cause destruction of the whole transformer. The comparison principle yields a natural assertion: Any rise of the density of heat sources always causes that the temperature will not decrease at any point. This confirms that the nonlinear mathematical model (1.1)-(1.2) of heat conduction has reasonable properties.

Re mark 4.1. We have $\alpha=0$ on $\partial \Omega \backslash \Gamma$. Setting $v=1$, we see by (2.5), Definition 2.1 and (2.6) that

$$
\begin{aligned}
\int_{\Gamma} \alpha u d s & =\int_{\partial \Omega} \alpha u d s=a(u ; u, 1)=F(1)=(f, 1)_{0, \Omega}+\langle g, 1\rangle_{0, \partial \Omega} \\
& =\int_{\Omega} f d x+\int_{\Gamma} \alpha u_{0} d s .
\end{aligned}
$$

Hence we observe an interesting fact that the average surface temperature rise $\vartheta_{\Gamma}$ on $\Gamma$ does not depend upon the type of nonlinearity of the heat conduction coefficients,

$$
\vartheta_{\Gamma} \equiv \frac{1}{\operatorname{meas} \Gamma} \int_{\Gamma}\left(u-u_{0}\right) d s=\frac{1}{\left.\alpha\right|_{\Gamma} \operatorname{meas} \Gamma} \int_{\Omega} f d x,
$$

provided $\left.\alpha\right|_{\Gamma}$ is constant.

Note that the total temperature flux on $\Gamma$ is also independent of the heat conduction coefficients (which follows from the Green theorem).

Remark 4.2. Numerical realization of the problem (1.1)-(1.2) can be obtained by the finite element method. The questions of the existence, uniqueness and convergence of discrete solutions are studied in [4, 13]. A discrete maximum principle is derived in [15]. For approximation of the curved boundary and numerical integration, see [6, 7, 24]. According to numerical tests [16], the hottest place of the magnetic core is in concave angles of the L- and T-joints (see Figure 3).

Acknowledgements. The authors wish to thank Prof. R. B. Kellogg of the University of Maryland for fruitful discussions and M. Lehtinen of the University of Jyväskylä for his help in carrying out the figures. The work was supported by grant No. A1019601 of the Grant Agency of the Academy of Sciences of the Czech Republic. This support is gratefully acknowledged. 


\section{References}

[1] L. Boccardo, T. Gallouët et F. Murat, Unicité de la solution de certaines équations elliptiques non linéaires, C. R. Acad. Sci. Paris Sér. I Math. 315 (1992), 1159-1164.

[2] L. Čermák and M. Zlámal, Transformation of dependent variables and the finite element solution of nonlinear evolution equations, Internat. J. Numer. Methods Engrg. 15 (1980), 31-40.

[3] P. Doktor, On the density of smooth functions in certain subspaces of Sobolev spaces, Comment. Math. Univ. Carolin. 14 (1973), 609-622.

[4] J. Douglas and T. Dupont, A Galerkin method for a nonlinear Dirichlet problem, Math. Comp. 29 (1975), 689-696.

[5] J. Douglas, T. Dupont and J. Serrin, Uniqueness and comparison theorems for nonlinear elliptic equations in divergence form, Arch. Rational Mech. Anal. 42 (1971), 157-168.

[6] M. Feistauer, M. Křížek and V. Sobotíková, An analysis of finite element variational crimes for a nonlinear elliptic problem of a nonmonotone type, EastWest J. Numer. Math. 1 (1993), 267-285.

[7] M. Feistauer and A. Ženíšek, Compactness method in finite element theory of nonlinear elliptic problems, Numer. Math. 52 (1988), 147-163.

[8] J. Franc ̊, Monotone operators. A survey directed to applications to differential equations, Appl. Math. 35 (1990), 257-301.

[9] H. Gajewski, K. Gröger and K. Zacharias, Nichtlineare Operatorgleichungen und Operatordifferentialgleichungen, Akademie-Verlag, Berlin, 1974.

[10] D. Gilbarg and N. S. Trudinger, Elliptic Partial Differential Equations of Second Order, Springer, Berlin, 1977.

[11] R. Glowinski, Numerical Methods for Nonlinear Variational Problems, Springer, New York, 1984.

[12] I. Hlaváček and M. Křížek, On a nonpotential and nonmonotone second order elliptic problem with mixed boundary conditions, Stability Appl. Anal. Contin. Media 3 (1993), 85-97.

[13] I. Hlaváček, M. Křížek and J. Malý, On Galerkin approximations of quasilinear nonpotential elliptic problem of a nonmonotone type, J. Math. Anal. Appl. 184 (1994), 168-189.

[14] R. Jensen, The maximum principle for viscosity solutions of fully nonlinear second order partial differential equations, Arch. Rational Mech. Anal. 101 (1988), 1-27.

[15] M. Kř́ížek and Q. Lin, On diagonal dominance of stiffness matrices in 3D, EastWest J. Numer. Math. 3 (1995), 59-69.

[16] M. Křížek and P. Neittaanmäki, Mathematical and Numerical Modelling in Electrical Engineering: Theory and Applications, Kluwer, Amsterdam, 1996.

[17] S. Larsson, V. Thomée and N. Y. Zhang, Interpolation of coefficients and transformation of the dependent variable in the finite element methods for the nonlinear heat equation, Math. Methods Appl. Sci. 11 (1989), 105-124.

[18] N. G. Meyers, An example of non-uniqueness in the theory of quasi-linear elliptic equations of second order, Arch. Rational Mech. Anal. 14 (1963), 177-179.

[19] J. Nečas, Les méthodes directes en théorie des équations elliptiques, Academia, Prague, 1967.

[20] - Introduction to the Theory of Nonlinear Elliptic Equations, Teubner, Leipzig, 1983. 
[21] M. H. Protter and H. F. Weinberger, Maximum Principles in Differential Equations, Prentice-Hall, Englewood Cliffs, N.J., 1967.

[22] W. Rudin, Real and Complex Analysis, McGraw-Hill, New York, 1966.

[23] J. Serrin, On the strong maximum principle for quasilinear second order differential inequalities, J. Funct. Anal. 5 (1970), 184-193.

[24] A. Ženíšek, Nonlinear Elliptic and Evolution Problems and Their Finite Element Approximations, Academic Press, London, 1990.

Michal Křižek and Liping Liu

Mathematical Institute

Academy of sciences

Žitná 25

CZ-115 67 Praha 1, Czech Republic

E-mail: Krizek@beba.cesnet.cz 